\title{
ESTENSIONE DELLA TEORIA QUANTISTICA DI CAMPO A TEORIA DI AUTOMA CELLULARE QUANTISTICO
}

\author{
Nota del m.e. GIACOMO MAURO D'ARIANO (*)
}

(Adunanza del 4 ottobre 2012)

SunTO. In questa nota riferisco brevemente sulla nuova teoria di campo basata sul paradigma informazionale. Il programma prosegue quello della derivazione assiomatica della teoria quantistica da principi di teoria dell'informazione, programma del quale presentai una memoria il 31 gennaio 2008. Il passaggio dalla teoria quantistica di sistemi generici alla teoria di campo si ottiene aggiungendo agli assiomi della teoria quantistica nuovi principi generali per la rete di interazioni di un insieme numerabile di sistemi quantistici. Tali principi si possono sintetizzare nella richiesta di minima complessità dell'algoritmo quantistico che descrive la legge fisica. Da principi generali quali omogeneità, isotropia, località, linearità, unitarietà della rete di interazioni, nonché dimensione minima del vettore di campo, si ricava la teoria di campo libera, ovvero i campi di Weyl, Dirac, e Maxwell. Ne deriva una descrizione del campo in termini di automa cellulare quantistico sul grafo di Cayley di un gruppo.

I vantaggi della nuova teoria sono molteplici. Ad esempio, vengono tautologicamente a mancare tutti i problemi che derivano dal continuo, quali le divergenze ultraviolette, il problema della localizzazione, e le violazioni della causalità. La meccanica stessa non viene assunta, bensì consegue dai principi, e la teoria è quantistica ab initio, ovvero non necessita della quantizzazione di una teoria di campo classica. La covarianza di Lorentz non è assunta, bensí consegue essa stessa nel limite relativistico di piccoli vettori d'onda, mentre la nozione di sistema di riferimento è riformulata in termini di rappresentazione irriducibile dell'automa quantistico. Infine, la nuova teoria, derivata da principi puramente matematici-e quindi con variabili adimensionali-contiene essa stessa al suo interno le unità di misura per distanza, massa, e tempo, e le unità sono date in termini di valori estremali delle variabili, in principio misurabili sperimentalmente. Dalla teoria ad automa emergono anche effetti con sapore relativistico-generale, quali un limite superiore alla massa a riposo della particella di Dirac (discendente dall'unitarietà

(*) Dipartimento di Fisica, Via Bassi 6, 27100 Pavia, Italy.

E-mail: dariano@unipv.it 
dell'automa), in corrispondenza del quale la relazione di dispersione diviene completamente piatta, in stretta analogia con la nozione di mini buco nero.

$* * *$

ABSTRACT. In the present note I will briefly report recent results on the novel field theory based on the information-theoretical paradigm. This program continues the previous one on the axiomatic derivation of quantum theory from information-theoretical principles, of which I presented a note on January 31st 2008. The passage from the quantum theory of abstract systems to the theory of fields is achieved by adding to the axioms of quantum theory new general principles for the network of interation among a denumerable set of quantum systems. Such principles can be synthesized with the requirement of minimal complexity of the quantum algorithm describing the physical law. From general principles such as homogeneity, isotropy, locality, linearity, and unitarity of the interaction network, along with minimal dimension of the field vector, one derives the free quantum field theory, namely Weyl, Dirac, and Maxwell fields. The principles lead to a description in terms of a quantum cellular automaton on the Cayley graph of a group. The advantages of the new theory are numerous. For example the theory tautologically solves all the problems originating from the continuum, such as the ultraviolet divergencies, the localization problem, and causality violations. The mechanics itself is not assumed, but it is a consequence of the principles, and the theory is quantum ab initio, namely it does not need quantization of a classical field theory. Lorentz covariance is not assumed, but follows itself from the principles, in the relativistic limit of small wavevectors. The notion of reference system is restated in terms of irreducible representation of the quantum automaton. Finally, the new theory, derived from purely mathematical principles-whence with adimensional variables-contains itself the units of measure for distance, time, and mass, which are given in terms of extremal values of the variables, which are in principle experimentally detectable. Also effects with GR flavor emerge from the automaton theory, such as an upper bound for the Dirac particle rest-mass (from the unitariety condition), where the dispersion relation becomes completely flat, in strict analogy with the notion of mini black hole.

\section{INTRODUZIONE}

Nell'adunanza del 31 gennaio 2008 presentai una memoria [1] su un mio lavoro in corso, iniziato nell'agosto 2003 , sulla fondazione assiomatica della Teoria Quantistica-la struttura teorica portante di tutta la fisica contemporanea. Il programma di assiomatizzazione ha raggiunto il risultato desiderato con due lunghi lavori pubblicati su Physical Review A $[2,3]$ in collaborazione con i miei ex allievi Paolo Perinotti e Giulio Chiribella ${ }^{1}$. Nel lavoro [3], la Teoria Quantistica è derivata da assiomi di

\footnotetext{
${ }^{1}$ Il lavoro [3] è stato selezionato dall' American Physical Society (APS) per il viewpoint di Časlav Brukner [4] (l'APS seleziona per un viewpoint sulla Physical Review circa un
} 
natura puramente informazionale. In sintesi, la Teoria Quantistica è una vera e propria teoria dell'informazione, e gli assiomi (dotati di interpretazione fisica a differenza degli assiomi Hilbertiani) sono principi generali di epistemologia, e riguardano la falsificabilità di una qualsiasi proposizione della teoria nella situazione di completo controllo sperimentale. La nuova formulazione assiomatica permette anche di dimostrare la maggior parte dei risultati chiave della teoria quantistica senza utilizzare spazi di Hilbert e operatori, conducendo ad un nuovo modo di comprendere la teoria.

Nella presente memoria, desidero invece parlare del passo successivo alla deduzione assiomatica della teoria quantistica, ovvero la riformulazione della parte meccanica della teoria. Ciò si ottiene derivando la teoria quantistica dei campi a partire da principi puramente informazionali. Questo programma realizza il paradigma informazionale profetizzato da John Archibald Wheeler e sintetizzato dalla frase del suo slogan: It from Bit-un mondo fisico fatto di bits. Con il senno di poi, oggi diciamo: un mondo fatto di qubits, ovvero "bit quantistici". Un mondo fatto da puro software, software senza hardware: Fisica come Informazione. Cosa vuol dire?

Si deve immaginare che non esistono atomi, ne' particelle, ne' fotoni, ma che questi sono patterns risultanti da una computazione su un calcolatore infinito quantistico ${ }^{2}$, come se la realtà fisica fosse un'enorme simulazione, ovvero tutto ciò che è fisico-inclusi spazio, tempo, massa, energia, gravitazione-emerge dalla computazione.

Le domanda più frequente che mi viene posta è: da che tipo di hardware è supportato il software se è la materia stessa che viene simulata? Di cosa sono fatti i qubits? Questa domanda non ha senso, e sorge perchè siamo abituati a pensare tutto in termini di materia o di energia. Ma il noto teorema di Malament [6] della teoria algebrica di campo dimostra che il concetto di particella è logicamente insostenibile: l'alternativa è la cosiddetta ontologia del campo [7]. Non dobbiamo dimenticare che il modo in cui immaginiamo la realtà deriva dalla teoria

lavoro su 2000). È in corso di pubblicazione un testo della Cambridge University Press, che sarà presentato prossimamente a questa Accademia.

${ }^{2}$ Del computer quantistico parlai in Accademia la prima volta il 18 novembre del 1998 nell'ambito di un ciclo di conferenze su Scienza e Tecnologie Avanzate, e suggerisco al lettore il relativo estratto per un breve presentazione divulgativa [5]. 
che ne abbiamo, e fra le varie teorie non falsificate dall'esperimento la scelta va alla teoria che deriva le osservazioni sperimentali dal minor numero di assunzioni. Il paradigma informazionale di Wheeler promette di derivare l'intera fisica dalla sola teoria quantistica, con l'aggiunta di poche semplici regole generali di semplicità dell'architettura del software. E queste nuove regole, insieme a quelle della teoria quantistica, risultano essere tutti principi metodologici alla base dello stesso approccio scientifico. Il vantaggio notevole è la possibilità di derivare la relatività stessa dalla teoria quantistica, aprendo la strada maestra alla riconciliazione fra le due teorie-la soluzione del problema più rilevante della fisica teorica contemporanea, nonchè chiave di volta della teoria della gravità.

Sostituire un mondo fatto di particelle che viaggiano in uno spaziotempo Minkowskiano con un cosmo evanescente fatto di pura informazione è indiscutibilmente un cambiamento di ontologia notevole, e di primo acchito il fisico teorico potrebbe essere riluttante ad adottare questo nuovo punto di vista. Ma le nozioni di spazio-tempo e di particella sono ontologicamente inconsistenti se guardate con gli occhi della fisica teorica contemporanea: la particella, oltrechè origine della schizofrenia ontologica del dualismo onda-corpuscolo, è solo uno stato "quantistico del campo", ovvero una mera regola di probabilità-una entità soggettiva nell'interpretazione della probabilità secondo Bayes e de Finetti.

Nel breve spazio di questa memoria riassumerò i risultati recenti della nuova teoria, illustrando come la teoria libera quantistica relativistica di campo emerga dalla computazione in termini di equazioni descriventi il flusso libero dell'informazione sulla rete computazionale e, insieme ad essa, emerga anche lo spazio-tempo e l'invarianza relativistica. Alcuni risultati preliminari su una derivazione euristica dell'equazione di Dirac nel paradigma informazionale sono stati presentati nella memoria del 16 Dicembre 2010 [8]. Per esposizioni divulgate si vedano anche i lavori $[9,10,11,12] .^{3}$

\footnotetext{
${ }^{3}$ I lavori $[11,12]$ sono derivati da due essays premiati dal Foundational Question Institute (FQXi).
} 


\section{I PRINCIPI GENERALI DELLA NUOVA TEORIA DI CAMPO}

La teoria quantistica è una teoria generale applicabile ad ogni tipo di sistema. Nella assiomatizzazione informazionale [3] la teoria è una teoria operazionale, ovvero una teoria probabilistica dotata di regole di connettività fra gli eventi. Matematicamente ciò corrisponde ad avere le probabilità congiunte dipendenti parametricamente da un circuito che descrive relazioni di input-output tra gli eventi. La nozione di evento viene "vestita" con i "sistemi" che rappresentano le connessioni fra gli eventi, e questi si connettono fra loro in circuiti aciclici chiusi. Matematicamente le regole di connessione sono formalizzate dalla categoria monoidale in teoria delle categorie. ${ }^{4} \mathrm{La}$ teoria quantistica, in quanto teoria operazionale, è quindi un'estensione della teoria delle probabilità, la quale è a sua volta un estensione della logica $[13,14]$ : in tal senso il programma di assiomatizzazione informazionale è la naturale continuazione del programma della quantum logic di von Neumann [15].

Nel framework operazionale si definiscono i seguenti assiomi per la teoria quantistica [3]:

1. Causalità

2. Discriminabilità locale

3. Discriminabilità perfetta

4. Atomicità della composizione

5. Compressione ideale

6. Purificazione

Per una esposizione divulgata rimando alla memoria [1], mentre per la formulazione matematica dei principi e la derivazione tecnica della teoria quantistica si veda il lavoro [3].

La teoria di campo libera in spazio piatto viene derivata aggiungendo gli assiomi seguenti, riguardanti la rete di interazione di un insieme numerabile di sistemi [16]:

\footnotetext{
${ }^{4}$ Per un esposizione dettagliata si veda [3].
} 
7. Minimalità della dimensione $s$ del sistema

8. Linearità

9. Unitarietà

10. Località

\section{Omogeneità}

12. Embeddabilità quasi-isometrica in spazio Euclideo

\section{Isotropia}

Come vedremo, in accordo all'assioma 7 di minimalità dimensionale i sistemi hanno dimensionalità è $s=2 .{ }^{5} \mathrm{~L}$ 'assioma 8 di linearità corrisponde a scrivere l'evoluzione del vettore di stato del qubit in termini di combinazione lineare dei sistemi che con esso interagiscono mediante matrici di transizione in $\mathbb{M}_{s}(\mathbb{C})$. L'assioma 9 di unitarietà si traduce in un insieme di equazioni che le matrici di transizione devono soddisfare. L'assioma 10 di località impone che l'insieme dei sistemi che interagiscono con un dato sistema (ovvero connessi da matrici di transizione non nulle) è uniformemente limitato. L'assioma 11 di omogeneità stabilisce che tale numero di sistemi interagenti è uguale per tutti i sistemi, che l'insieme delle matrici di transizione è lo stesso per tutti i sistemi, ed infine che tutte le loop di catene di sistemi interagenti sono le stesse a partire da ogni sistema. Degli assiomi 12 e 13 parlerò nel seguito.

Dagli assiomi 10 e 11 discende che le connessioni fra i sistemi interagenti sono descritti dal grafo di Cayley di un gruppo $G$, ovvero un grafo direzionato e con links colorati, dove i siti rappresentano elementi del gruppo $G$, i links sono in corrispondenza con coppie di elementi, il colore del link è in corrispondenza con un generatore del gruppo, e la freccia sul link rappresenta l'operazione del generatore del gruppo sull'elemento alla coda della freccia con risultato l'elemento alla punta della freccia. Quindi il grafo delle interazioni è in corrispondenza con una particolare presentazione $G=\left\langle h_{1}, h_{2}, \ldots \mid r_{1}, r_{2}, \ldots\right\rangle$ del gruppo $G$

\footnotetext{
${ }^{5}$ Nella teoria interagente o se si considera l'equivalente di più particelle, il qubit diviene un campo Fermionico.
} 
mediante generatori $h_{1}, h_{2}, \ldots$, e relatori $r_{1}, r_{2}, \ldots$ Ad ogni generatore $h$ è associata una matrice di transizione in $\mathbb{M}_{2}(\mathbb{C})$.

Si dimostra che gli assiomi 8 e 9 danno luogo a un operatore unitario sullo spazio di Hilbert $\ell^{2}(G) \otimes \mathbb{C}^{s}$ di singolo sistema quantistico tensorizzato lo spazio della rappresentazione right-regular di quadrato sommabile del gruppo $G$. La condizione di unitarietà si traduce in un insieme non-banale di condizioni bilineari sulle matrici di transizione.

La Geometric Group Theory. La suddetta costruzione matematica, che segue direttamente dagli assiomi 7-11 collega la nuova teoria di campo ad una branca della matematica pura contemporanea: la Geometric Group Theory. L'idea alla base, dovuta Mikhail Gromov, è di studiare le proprietà del gruppo assegnato dalla presentazione $G=\left\langle l_{1}, l_{2}, \ldots\right| r_{1}$, $\left.r_{2}, \ldots\right\rangle$ in termini delle proprietà dello spazio metrico $M(G)$ nella quale il grafo di Cayley si può embeddare quasi-isometricamente, ${ }^{6}$ conducendo ad una geometrizzazione del problema gruppale. Ad esempio, restringendoci a gruppi infiniti che rappresentano il caso di interesse, un teorema dovuto a Gromov dimostra che lo spazio metrico $M(G)$ in cui si embedda il gruppo è Euclideo se e solo se il gruppo $G$ è virtualmente Abeliano, ${ }^{7}$ altrimenti $G$ è non virtualmente Abeliano e $M(G)$ è iperbolico. La logica è semplicemente il confronto delle crescite dei volumi della palla ${ }^{8}$ di raggio $r$ in funzione di $r$, rispettivamente per $M(G)$ e $G$, e tale crescita è polinomiale se $M(G)$ è Euclideo e $G$ è virtualmente Abeliano, mentre è esponenziale se $M(G)$ è iperbolico e $G$ è infinito e non virtualmente Abeliano.

Il lettore si chiederà cosa centra tutto ciò con la teoria di campo: la risposta è che lo spazio metrico $M(G)$ nella nuova teoria risulta essere lo spazio fisico emergente dalle interazioni fra i sistemi. Il postulato

\footnotetext{
${ }^{6}$ La metrica sul grafo è la counting-metric intera, mentre quella sullo spazio metrico è reale. Il termine embedding quasi isometrico vuol dire che la discrepanza fra le due metriche è uniformemente limitata, ad esempio è data dal fattore $\sqrt{d}$, dove $d$ è la dimensione dello spazio metrico. Inoltre l'embedding deve anche essere quasi-onto, ovvero ogni punto dello spazio metrico dista da un sito del grafo non più di una costante.

${ }^{7}$ Un gruppo si dice virtualmente Abeliano se contiene un sottogruppo Abeliano di indice finito.

${ }^{8} \mathrm{Il}$ volume della palla di raggio $r$ nel grafo è data dal numero di punti che distano non più di $r$ steps da un punto qualunque del grafo.
} 
11 restringe la teoria a quella su spazi Euclidei. Tale restrizione è fisicamente motivata dal fatto che nel caso iperbolico l'informazione trasmessa decresce esponenzialmente con la distanza (il caso di gruppo iperbolico sarà comunque analizzato in futuro).

Il postulato dell'isotropia. Resta infine il postulato di isotropia. Innanzitutto si richiede che nell'embedding quasi-isometrico di $G$ in $M(G)$ la sfera unitaria sul grafo sia embeddata nella sfera unitaria in $M(G)$. Viene richiesta quindi l'esistenza di un gruppo $P$ di permutazioni dei generatori di $G$ transitivo che agisce come automorfismo del grafo di Cayley, ed avente una rappresentazione (generalmente proiettiva) unitaria in dimensione $s$. Si richiede quindi che l'evoluzione unitaria sia invariante sotto l'azione congiunta del gruppo $P$ sul grafo di Cayley e sullo spazio $s$-dimensionale dei vettori di stato del singolo sistema.

\section{LA DERIVAZIONE DELLA TEORIA QUANTISTICA DI CAMPO LIBERA NEL LIMITE RELATIVISTICO}

Nel lavoro [16] con Paolo Perinotti ci restringiamo al caso di gruppo $G$ Abeliano, anziché al più generale caso virtualmente Abeliano. ${ }^{9}$ In questo lavoro dimostriamo che l'unico grafo di Cayley embeddabile quasi-isometricamente in $\mathbb{R}^{3}$ che permette di soddisfare i principi 7-13 è il reticolo BCC (body centered cubic). Nessuno degli altri reticoli permette di soddisfare le condizioni di unitarietà per dimensione $s=2$ dello spazio di Hilbert di singolo sistema, mentre per dimensione $s=1$ (equivalente a campo scalare) sussiste solo il caso di evoluzione banale. Inoltre, esistono solo due possibili trasformazioni unitarie (a meno di cambi di rappresentazione locali e di particolari traslazioni nello spazio dei vettori d'onda), ovvero esistono due automi cellulari quantistici che soddisfano i principi, e i due automi sono connessi fra loro dalla simmetria di parità. Si noti che il concetto di vettor d'onda è ben definito, essendo il gruppo con Cayley graph BCC Abeliano. Nel cosiddetto limite relativistico di vettori d'onda piccoli (ovvero vicino al centro della

\footnotetext{
${ }^{9} \mathrm{Da}$ studi tuttora in corso sembra che la restrizione al caso Abeliano non sia rilevante per quanto segue nel testo. L'utilizzazione dei gruppi virtualmente Abeliani riduce l'analisi al caso scalare per un sottogruppo Abeliano di indice 4, e permette una derivazione unificata degli automi di Weyl, Maxwell, e Dirac (si veda il seguito).
} 
zona di Brillouin) si trova che l'evoluzione unitaria è data esattamente dall'equazione di Weyl (i due automi corrispondono all'evoluzione per spinore rispettivamente sinistrorso e destrorso). Con questi due automi elementari di Weyl si possono ottenere automi composti in due modi: mediante somma diretta e mediante prodotto tensore dell'operatore unitario con il suo aggiunto. ${ }^{10} \mathrm{Nel}$ caso della somma diretta per preservare il principio di località è necessario usare un accoppiamento indipendente dal vettori d'onda nei blocchi fuori diagonale. Dalla composizione degli automi, per ognono dei due possibili automi di Weyl si ottiene un automa con dimensione $s=4$.

In sintesi, si ha:

Accoppiamento in somma diretta [16]. Si hanno due nuovi automi che nel limite relativistico danno entrambi l'equazione di Dirac. Questi due automi sono connessi da CPT, e la violazione della simmetria denota la rottura della simmetria di Lorentz. Il parametro di accoppiamento nella somma diretta gioca il ruolo della massa a riposo $m$, e l'unitarietà comporta che i blocchi sulla diagonale siano riscalati di una quantità $n$, con $n^{2}+m^{2}=1$, in modo da mantenere la normalizzazione delle righe dell'operatore, e il fattore di riscalamento gioca il ruolo della velocità della luce. Ne deriva che per soddisfare l'unitarietà la massa a riposo $m \leq 1$ è limitata superiormente, e che all'approssimarsi al valore $m=1$ la relazione di dispersione dell'automa diventa piatta $(n=0)$. Un tale comportamento ricorda quello di un mini buco nero, laddove la massa della particella è limitata superiormente dall'uguaglianza della lunghezza d'onda Compton con il raggio di Schwarzschild. Si noti infine che la massa inerziale nel contesto della nuova teoria viene introdotta senza dover utilizzare la definizione meccanica circolare basata sulla la legge di Newton [17]: è questo un vantaggio fondazionale notevole, che deriva dal fatto che la teoria è quantum ab initio. Per altri aspetti riguardanti l'automa di Dirac, si vedano anche i lavori $[18,19,20]$.

\footnotetext{
${ }^{10} \mathrm{Nel}$ caso del prodotto tensore con lo stesso operatore si ottiene l'evoluzione di più particelle identiche, mentre il tensore con l'aggiunto dà luogo ad un nuovo automa. Invece, l'unico modo di costruire un automa per somma diretta che preserva il principio di località è quello di sommare a blocchi l'operatore unitario con il suo aggiunto, e usare un accoppiamento indipendente dal vettor $\mathrm{d}$ 'onda nei blocchi fuori diagonale.
} 
Accoppiamento a prodotto tensore [21]. Si hanno due nuovi automi con lo stesso limite relativistico, corrispondente ad un campo vettoriale che evolve secondo l'equazione di Maxwell, e un campo scalare costante. Il campo vettoriale non è fatto di Bosoni, bensì di coppie di Fermioni entangled. La statistica che ne risulta è comunque indistinguibile da quella Fermionica, e non sono rilevabili effetti di saturazione Fermionica anche con potenze di radiazione dell'ordine del Petawatt, come le potenze ottenibili da un laser Vulcan laser. L'equazione di Maxwell risulta debolmente distorta. Si hanno principalmente due effetti: 1) esiste una componente longitudinale della polarizzazione, e il vettore di Pointing, la velocità di gruppo e il vettor d'onda hanno direzioni leggermente diverse; 2) la velocità di gruppo differisce di poco da $c$, dipendendo dalla lunghezza d'onda e dalla direzione. L'effetto 1 è praticamente non rilevabile sperimentalmente, mentre l'effetto 2 si accumula durante la propagazione, e può essere rivelato come shift di pacchetti di radiazione a frequenze diverse provenienti da un evento cosmico ai limiti dell'universo visibile. La teoria a Fermioni della radiazione era stata già considerata da De Broglie, ma fu in seguito scartata perchè non obbediva alla statistica di Bose-Einstein.

\subsection{Unità di misura all' interno della teoria}

Il bound sul valore della massa permette anche di stabilire le unità di misura all'interno della teoria. Infatti, una teoria fondata su assiomi necessariamente matematici (sebbene dotati di interpretazione fisica), avrà necessariamente variabili solo adimensionali, che potranno essere scritte come rapporto fra il valore fisico misurato e il valore campione. Nella teoria ad automa quantistico la variabile tempo adimensionale è intera, e si scriverà quindi come il rapporto fra il tempo fisico e il valore campione $t_{P}$. Analogamente per la distanza spaziale in termini della distanza campione $x_{P}$. Invece, la massa divisa per la massa campione $m_{P}$ è un numero compreso fra 0 e 1 . La massa campione $m_{P}$ è quindi il valore massimo della massa di una particella, e può essere misurato, in principio, mediante determininazione sperimentale dell'indice di rifrazione di massa (come già accennato, a causa dell'unitarietà $n^{-1}$ giuoca il ruolo di indice di rifrazione). Dal confronto della teoria dell'automa con l'usuale teoria quantistica di campo nel limite relativistico si ricavano le relazioni 
$c=x_{P} / t_{P}$ e $\hbar=m_{P} x_{P} c$, dove $c$ rappresenta la velocità della luce, e $\hbar$ la costante di Planck. La teoria di automa cellulare quantistico prevede per la radiazione una velocità che nel limite relativistico dipende linearmente dal vettor d'onda. Da tale dipendenza si può determinare ad esempio $x_{P}$, mentre $t_{P}$ sarà dato da $x_{P} / c$. Quindi, conoscendo il valore di $\hbar$ e di $c$, dalla determinazione sperimentalmente di $x_{P}$ oppure di $m_{P}$ otteniamo i valori delle tre misure campione. Un ordine di grandezza di tali valori può essere ottenuto assumendo ad esempio che l'accelerazione gravitazionale dovuta a una massa $m_{P}$ a distanza minima $x_{P}$ dia la costante di gravitazione $G$ secondo la legge di Newton, nel qual caso $m_{P}=2.176 * 10^{-8} \mathrm{~kg}$ è la massa di Planck, $x_{P}=1,616 * 10^{-35} \mathrm{~m}$ è la lunghezza di Planck e $t_{P}=5.391 * 10^{-44}$ s è il tempo di Planck.

\subsection{Bontà del limite relativistico}

La teoria di campo ad automa cellulare quantistico è un'estensione della teoria quantistica di campo a scale molto piccole, dell'ordine della scala di Planck, e contiene la teoria di campo quantistica usuale come limite relativistico di piccoli vettor d'onda (come abbiamo visto, per "piccoli" si intende rispetto al vettor d'onda di Planck) ${ }^{11}$ La teoria rappresenta in modo molto fedele la teoria di campo usuale nel limite relativistico: ad esempio si calcola [16] che l'evoluzione dell'automa per un protone relativistico è indistinguibile da quella di Dirac, con qualunque tecnologia, per un evoluzione temporale di milioni di anni, mentre effetti alla portata della tecnologia attuale sono visibili su evoluzioni di miliardi di anni.

\section{COVARIANZA DI LORENTZ NELLA TEORIA DI CAMPO AD AUTOMA CELLULARE, E NOZIONE DI PARTICELLA}

Uno dei punti concettualmente più rilevanti della nuova teoria di campo ad automa è che essa permette di derivare la teoria di campo senza assumere la meccanica, e quindi nemmeno l'invarianza di Lorentz, ma come conseguenza solo di principi molto generali, come quelli elencati nella Sezione 2. Inoltre, oltre che essere quantistica ab initio-ovvero

\footnotetext{
${ }^{11}$ Ad esempio una particella di un ultra bigh energy cosmic ray ha vettor d'onda $10^{-8}$ volte il valore del vettor d'onda di Planck.
} 
non necessita della quantizzazione della teoria classica-la nuova teoria, essendo discreta, elimina per definizione tutti i problemi dalla teoria di campo usuale derivanti dal continuo: in particolare, oltre alle divergenze ultraviolette, il problema della localizzazione della particella. Il calcolo del propagatore come path integral è ben definito, ed è stato calcolato analiticamente per il caso di Dirac in una dimensione spaziale [22] e per quello di Weyl in due dimensioni [23]. L'invarianza di Lorentz è riottenuta esattamente nel limite di piccoli vettori d'onda.

Cosa succede del principio di relatività alla scala discreta? Accade che il gruppo di Lorentz diventa non lineare [24], ed avvengono nuovi fenomeni, quali ad esempio, il fenomeno della relative locality [25]-fenomeno previsto una quindicina di anni fa da Amelino-Camelia e Smolin-in base al quale non solo la simultaneità temporale di eventi, ma anche la loro vicinanza spaziale dipendono dall'osservatore. Nel contesto della teoria ad automa il cambiamento di sistema di riferimento è definito come un cambiamento di rappresentazione dell'automa, corrispondente a rimappare il vettor d'onda $k \mapsto k^{\prime}(k)$ e corrispondentemente la frequenza $\omega(k) \mapsto \omega^{\prime}\left(k^{\prime}\right)$. È interessante notare che per massa $m=0$ sopravvive il concetto di particella come invariante del gruppo di Poincaré (nel nostro caso il gruppo di Poincaré nonlineare), e che ad esempio, per l'automa di Dirac si hanno quattro particelle (e rispettive antiparticelle) diverse in corrispondenza di quattro zone invarianti all'interno della zona di Brillouin del BCC. Infine, se la massa a riposo $m$ è nonnulla, nel caso ultrarelativistico le trasformazioni nonlineari hanno anche una dipendenza dal boost della massa a riposo, e ciò forza ad estendere il gruppo di invarianza al gruppo di de Sitter [26], mentre la simmetria di Lorentz è ritrovata all'ordine $m^{2}$.

\section{Ringraziamenti}

Desidero vivamente ringraziare la collaborazione intensa, costante, e cruciale del Prof. Paolo Perinotti, e il notevole lavoro dei Dott. Alessandro Bisio e Alessandro Tosini. Desidero anche ringraziare l'incoraggiamento e il sostegno dei membri dell'Accademia prof. Attilio Rigamonti e Prof. Giorgio Goggi. Questa ricerca è finanziata dalla Templeton Fundation (project ID\# 43796 A Quantum-Digital Universe), e dalla Fondazione della Banca del Monte di Lombardia. 


\section{REFERENCES}

[1] G. M. D'Ariano, Da quali principi discende la Meccanica Quantistica?, Rendiconti Istituto Lombardo - Accademia di Scienze e Lettere 14223 (2008)

[2] G. Chiribella, G. M. D'Ariano, P. Perinotti, Probabilistic Theories with Purification, Phys. Rev. A 81062348 (2010)

[3] G. Chiribella, G. M. D'Ariano, P. Perinotti, Informational derivation of Quantum Theory, Phys. Rev A 84012311 (2011)

[4] Č. Brukner, Questioning the rules of the game, Physics 455 (2011).

[5] G. M. D'Ariano, and C. Macchiavello, Introduzione alla teoria dell'Informazione Quantistica, Rendiconti Istituto Lombardo Accademia di Scienze e Lettere 137289 (2003)

[6] H. Halvorson and R. Clifton, No Place for Particles in Relativistic Quantum Theory?, Phil. Sc. 691 (2002)

[7] M. Kuhlmann, What is Real?, Sc. Am. 32 August, (2013) 1

[8] G. M. D'Ariano, La Fisica come Informazione: l'universo è un grande automa quantistico, Rendiconti Istituto Lombardo - Accademia di Scienze e Lettere 144253 (2010)

[9] G. M. D'Ariano, A Quantum Digital Universe, Il Nuovo Saggiatore 2813 (2012)

[10] G. M. D'Ariano, L'Univers est-il un ordinateur quantique?, La Recherche, Juillet-Aout 2014, n.489, pag.48

[11] G. M. D'Ariano, It from Qubit, in A. Aguirre et al. (eds.), It From Bit or Bit From It?, The Frontiers Collection (Springer 2015)

[12] G. M. D'Ariano, Quantum-Informational Principles for Physics, in A. Aguirre et al. (eds.), Questioning the Foundations of Physics, The Frontiers Collection (Springer 2015) 
[13] R. T. Cox, The Algebra of Probable Inference (Johns Hopkins University Press, Baltimore 1961)

[14] E. T. Jaynes, Probability Theory: The Logic of Science (Cambridge University Press, Cambridge 2003)

[15] G. Birkhoff and J. von Neumann, The logic of quantum mechanics, Ann. Math. 37823 (1936)

[16] G. M. D'Ariano, P. Perinotti, Derivation of the Dirac Equation from Principles of Information processing, Phys. Rev. A 90062106 (2014)

[17] M. Jammer, Concepts of Mass in Contemporary Physics and Philosophy, (Princeton University Press, Princeton 1999)

[18] A. Bisio, G. M. D'Ariano, A. Tosini, Quantum Field as a Quantum Cellular Automaton: the Dirac free evolution in one dimension, Ann. Phys. 354244 (2015)

[19] G. M. D'Ariano, F. Manessi, P. Perinotti, A. Tosini, The Feynman problem and Fermionic entanglement: Fermionic theory versus qubit theory Int. J. Mod. Phys. A 291430025 (2014)

[20] G. M. D'Ariano, F. Manessi, P. Perinotti, A. Tosini, Fermionic computation is non-local tomographic and violates monogamy of entanglement, EPL 10720009 (2014)

[21] A. Bisio, G. M. D'Ariano, P. Perinotti, Quantum Cellular Automaton Theory of Light, arXiv: 1407.6928

[22] G. M. D'Ariano, N. Mosco, P. Perinotti, A. Tosini, Path-integral solution of the one-dimensional Dirac quantum cellular automaton, Phys. Lett. A 3783165 (2014)

[23] G. M. D'Ariano, N. Mosco, P. Perinotti, A. Tosini, Discrete Feynman propagator for the Weyl quantum walk in $2+1$ dimensions, EPL 10940012 (2015)

[24] A. Bisio, G. M. D'Ariano, P. Perinotti, Lorentz symmetry for 3d Quantum Cellular Automata, arXiv:1503.01017 
[25] A. Bibeau-Delisle, A. Bisio, G. M. D'Ariano, P. Perinotti, A. Tosini, Doubly-Special Relativity from Quantum Cellular Automata, EPL 10950003 (2015)

[26] A. Bisio, G. M. D'Ariano, P. Perinotti, in preparazione. 